Oleksandr Novostroinyi

V.I. Samusia, research supervisor

V.O. Lapina, language adviser

National Mining University, Dnipro, Ukraine

\title{
Electrical Power Industry
}

One of the most prominent sources that changed the life of the whole world was the discovery of the most efficient energy source - electricity.

From the scientific point of view, the electricity is a particular set of physical phenomena which is characterized by the presence and the distinctive flow of electric charge. It is created when the small particles - electrons move between the atoms. This process creates an electric current. This current is used to energize different kinds of equipment.

In our modern world electricity is used for industry and agriculture, communication and transportation. We use electrical power for heating, cooling and lighting our houses, for cooking food, and for numerous devices and gadgets such as TV-sets, computers and smart phones. Electrical power industry can be fair enough called a backbone of the modern industry and everyday life.

The electric power industry is commonly split up into four processes. These are electricity generation such as a power station, electric power transmission, electricity distribution and electricity retailing.

Electricity generation is the process of generating electric power from sources of primary energy. For electric utilities, it is the first process in the delivery of electricity to consumers. Electricity is most often generated at a power station by electromechanical generators, primarily driven by heat engines fuelled by combustion or nuclear fission but also by other means such as the kinetic energy of flowing water and wind.

Electric power transmission is the bulk movement of electrical energy from a generating site, such as a power plant, to an electrical substation. The interconnected lines which facilitate this movement are known as a transmission network. This is distinct from the local wiring between high-voltage substations and customers, which is typically referred to as electric power distribution. The combined transmission and distribution network is known as the "power grid" in North America, or just "the grid". In the United Kingdom, the network is known as the "National Grid".

Electric power distribution is the third stage in the delivery of electric power. It carries electricity from the transmission system to individual consumers. Distribution substations connect to the transmission system and lower the transmission voltage to medium voltage ranging between $2 \mathrm{kV}$ and $35 \mathrm{kV}$ with the use of transformers. Primary distribution lines carry this medium voltage power to distribution transformers located near the customer's premises. Distribution transformers again lower the voltage to the utilization voltage of household appliances and typically feed 
several customers through secondary distribution lines at this voltage. Commercial and residential customers are connected to the secondary distribution lines through service drops. Customers demanding a much larger amount of power may be connected directly to the primary distribution level or the subtransmission level.

Electricity retailing is the final sale of electricity from generation to the end-use consumer. This is the fourth step in the electricity delivery process: after generation, transmission and distribution.

The amount of energy used by the domestic consumer, and thus the amount charged for, is measured through an electricity meter that is usually placed near the input of a home to provide easy access to the meter reader. Customers are usually charged a monthly service fee and additional charges based on the electrical energy (in $\mathrm{kWh}$ ) consumed by the household or business during the month. Commercial and industrial consumers normally have more complex pricing schemes. These require meters that measure the energy usage in time intervals (such as a half-hour) to impose charges based on both the amount of energy consumed and the maximum rate of consumption, i.e. the maximum demand, which is measured in $\mathrm{kVA}$

The provision of electricity services was generally the responsibility of electric companies or municipal authorities who either set up their own departments or contracted the services from private entrepreneurs.

In many countries, electric power companies own the whole infrastructure from generating stations to transmission and distribution infrastructure. For this reason, electric power is viewed as a natural monopoly. The industry is generally heavily regulated, often with price controls and is frequently government-owned and operated.

Besides the obvious advantages that electrical power brings to our life there is a definite set of threats that this modern technology causes. The process of electricity generation on different kinds of power stations often is not so harmless to the nature. One of the most efficient but dangerous means of electricity generation is a nuclear power station. The disastrous catastrophes in Chernobyl and Fukusima showed us how dangerous nuclear power is. Though, this is one of the most effective ways to generate electricity for the needs of the society.

The process of nature friendly electricity generation has been developing greatly these days. Wind power, solar power and the power of the ocean are used to generate safe and cheap electricity that will be able to bring our life to the next level of evolution.

Although electrical power has become the essential necessity for the modern society, humanity must wisely use natural resources and develop ways of producing electricity in order to protect people and nature. That will lead the society to a new level of developing and living. 